\title{
Criyoglobulinemic Vasculitis Secondary to Multiple Myeloma
}

M Kavala ${ }^{1}$, AS Karadag ${ }^{1}$, E Ozturk $^{2}$, I Zindanci ${ }^{1}$, B Oman ${ }^{1}$, Z Turkoglu ${ }^{1}$, E Zemheri ${ }^{3}$, E Ozlu ${ }^{1}$, FT Demir $^{4}$

\begin{abstract}
Criyoglobulins are immunoglobulins that precipitate at low temperatures and dissolve at higher temperatures. The existence of cryoglobulins in serum is termed cryoglobulinaemia. Although, the patients with cryoglobulinaemia or cryoglobulinemic vasculitis, the consequence of cryoglobulinaemia, have typical symptoms related to the presence of cryoglobulins, may patients with cryoglobulinaemia remain asymptomatic. Criyoglobulinaemia a systemic vasculitis involving primarily small vessels, can be associated with infections, lymphoproliferative malignancies or autoimmune diseases. Type 1 criyoglobulins can be found in patients with, lymphoproliferative disorders, such as Waldernström macroglobulinaemia or multiple myelom, while mixt criyoglobulins are detected in patients with autoimmune diseases, lymphoproliferative disorders or infections, most commonly hepatitis $\mathrm{C}$ virus. We present a 38 -year-old man with criyoglobulinemic vasculitis secondary to multiple myeloma. The patient was admitted to hospital with one year history of cutaneous purpura, aggrevated with cold, on his legs and ears accompanied by arthralgia. Skin biopsy revealed leukocytoclastic vasculitis. There was serum cryoglobulinaemia and serum immunofixation electropheresis showed a IgG kapa monoclonal gamapathy. Contrasted computed tomography evidenced osteolytic lesions on his 4th rip, biopsy of which demonstrated a plasmocytoma. The bone marrow biopsy yielded $90 \%$ of kapa monoclonal plasma cells verifying the diagnosis of multiple myeloma.
\end{abstract}

Keywords: Criyoglobulinemic vasculitis, multiple myeloma, plasmositoma

From: ${ }^{1}$ Department of Dermatology, Medeniyet University, Goztepe Training and Research Hospital, Istanbul, Turkey. ${ }^{2}$ Department of Hematology, Medeniyet University, Goztepe Training and Research Hospital, Istanbul, Turkey. ${ }^{3}$ Department of Pathology, Medeniyet University, Goztepe Training and Research Hospital, Istanbul, Turkey. ${ }^{4}$ Department of Dermatology, Sisli Hamidiye Etfal Training and Research Hospital, Istanbul, Turkey

Correspondence: Dr FT Demir, Department of Dermatology, Sisli Hamidiye Etfal Training and Research Hospital, Sisli, Istanbul, 34371, Turkey. E-mail: filizsvet@yahoo.com 


\section{INTRODUCTION}

A case of cryoglobulinemic vasculitis secondery to multiple myeloma was reported, characterized by cutaneous purpura, aggrevated by cold, and leucocytoclastic vasculitis along with blood positivity to monoclonal IgG cryoglobulin and features of multiple myeloma. This report illustrates the importance of considering underlying diseases in any patient with cryoglobulinemic vasculitis.

\section{CASE REPORT}

A 38-years-old man presented with a year history of purplish eruption on his lower extremities and ears, aggrevated by cold.His past medical history includes Raynaud phenomenon and arthralgia. Examination revealed non blanching,discreata 1-8 mm purpuric lesions and some confluent hemarhages on both lower extremities extending to buttocks (Fig1, 2). Similar morphological lesions were evident in his ears (Fig3). There were seen hyperkeratosis, acanthosis of epidermis, vascular dilatation and accumulation of eosinophilic material in lumen of vessels, perivascular lymphocytes in dermis on skin biopsy. (Fig 4a)

Eosinophilic material in lumen of vessels was positively stained with periodic acid-schiff. (Fig 4b). A skin biopsy was consistent with a diagnosis of leucocytoclastic vasculitis,

Investigations, including complete blood count, liver and renal functions, syphilis, hepatitis $\mathrm{B}$ and $\mathrm{C}$ viruses, HIV, Epstein Barr virus and cytomegalovirus serologies and antinuclear antibody, rheumatoid factor and antineutrophil cytoplasmic antibodies were negative or normal. Complement $\mathrm{C} 3$ was normal while $\mathrm{C} 4$ was reduced to $1.8 \mathrm{mg} / \mathrm{dl}(\mathrm{N}$ : $16.0-$ $38.0 \mathrm{mg} / \mathrm{dl}$ ). Testing for cryoglobulinemia was positive with $\mathrm{M}$ spike in the gamma region on protein electrophoresis. Serum immunofixation electrophoresis showed a IgG kappa monoclonal gammopathy. The patient was investigated for lympoproliferative disorders 
because of the monoclonal nature of the cryoglobulin. A computed tomography scan of the chest evidenced osteolytic lesions on the 4th rib, biopsy of which demonstrated a plasmacytoma. The bone marrow biopsy yielded $90 \%$ of kappa monoclonal plasma cells verifying the diagnosis of multiple myeloma (Fig 5a, b). Based on the clinical and histological findings and the presence of monoclonal IgG cryoglobulin in serum, a diagnosis of cryoglobulinemic vasculitis secondery to multiple myeloma was made, and the patient was discharged to the hematology clinic.

\section{DISCUSSION}

Cryoglobulins are immunoglobulins that precipitate as serum is cooled below core body temperatures (1). Criyoglobulinemia, the existence of cryoglobulins in serum, is an immune complex-mediated systemic vasculitis involving mostly small, but sometimes larger vessels, and can be associated with infections, autoimmune disorders, and malignancies. The most common cause is infection with hepatitis $\mathrm{C}$ virus (2-5). Cryoglobulinemic vasculitis is the consequence of cryoglobulinemia(3). The patients with cryoglobulinemia or cryoglobulinemic vasculitis have symptoms related to the presence of cryoglobulins. The percentage of patients who develop symptoms varies from $2 \%$ to $50 \%$ (6). Purpura, arthralgia, weakness, peripheral neuropathy, and acute nephritic or nephrotic syndrome are commonly seen in cryoglobulinemic vasculitis (2-5). The development of cryoglobulinemic symptoms is affected by age, underlying illness, and the characteristiesof the cryoglobulins (6).

Type 1 cryoglobulins can be found in patients with lymphoproliferative disorders, such as Waldenström's macroglobulinemia or multiple myeloma, while mixed cryoglobulins are detected in patients with autoimmune disorders, lymphoproliferative disorders or chronic 
infections. When no underlying disease is present, this is called essential or idiopathic cryoglobulinemia (1).

Cutaneous purpura is the most characteristic manifestation of cryoglobulinemic vasculitis(6), and serum complement factor 4 levels are usually low or below detection(7). Cryoglobulinemic vasculitis should be diagnosed in the presence of clinical signs of the vasculitis and cryoglobulinemia, and histologic proof of an immune complex-mediated vasculitis.

We report a man with cryoglobulinemic vasculitis due to multiple myeloma. Our case illustrates the importance of identifying the presence of underlying diseases in any patient with cryoglobulinemic vasculitis so that treatment can be optimized to treat the vasculitic process.

\section{AUTHORS' NOTE}

The paper has not been published or submitted for publication elsewhere. All authors have contributed significantly, and all authors are in agreement with the content of the manuscript. There is no conflict of interest among all authors. 


\section{REREFERENCES}

1. Brouet J,Clauvel JP,Danon F,Klein M,Seligmann M. Biologic and clinical significance of cryoglobulins:a report of 86 cases. Am J Med 1974; 57: 775-88.

2. Ramos-CasaLs M,Stone JH,Cid MC, Bosch X. The cryoglobulinaemias. Lancet 2012; 379: $348-60$.

3. Lamprecht P,Gause A,Gross WL.Cryoglobulinemic Vasculitis.Am Col of Rheum $1999 ; 42: 2507-16$.

4. Rieu V,Cohen P,Andre $\mathrm{MH}$ et al.Characteristics and outcome of 49 patients with symptomatic cryoglobulinaemia.Br Soc of Rheum 2002; 41: 290-300.

5. Kallemuchikkal U,Gorevic PD.Evaluation of Cryoglobulins.Arch Pathol Lab Med.1999;123:119-125.

6. Trejo O,Ramos-Casals M,Garcia -Carrasco $\mathrm{M}$ et al.Cryoglobulinaemia:study of etiologic factors and clinical and immnulogical features in 443 patients from a single center.Medicine(Baltimore) 2001;80:252-62.

7. Sargur P,White P,Egner W.Cryoglobulin evaluation:best practice?Ann Clin Biochem 2010; 47: 8-16. 


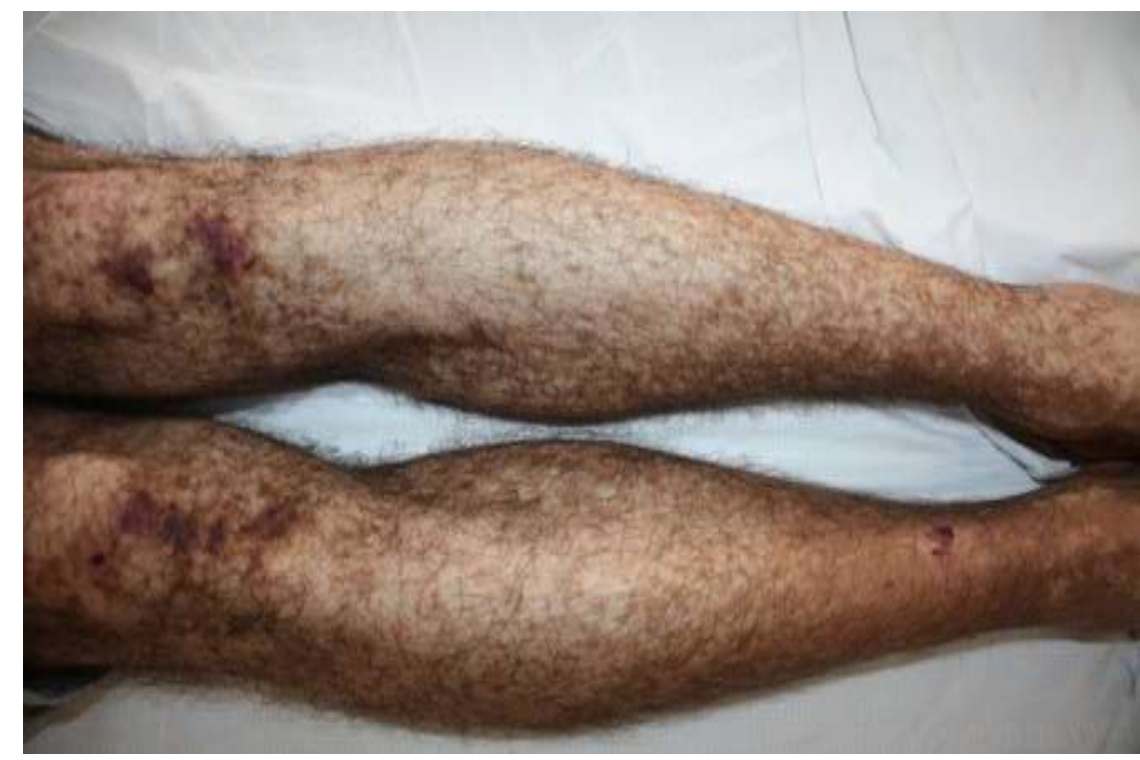

Fig 1: Purpuric lesions and some confluent hemorrhages on the lower extemities

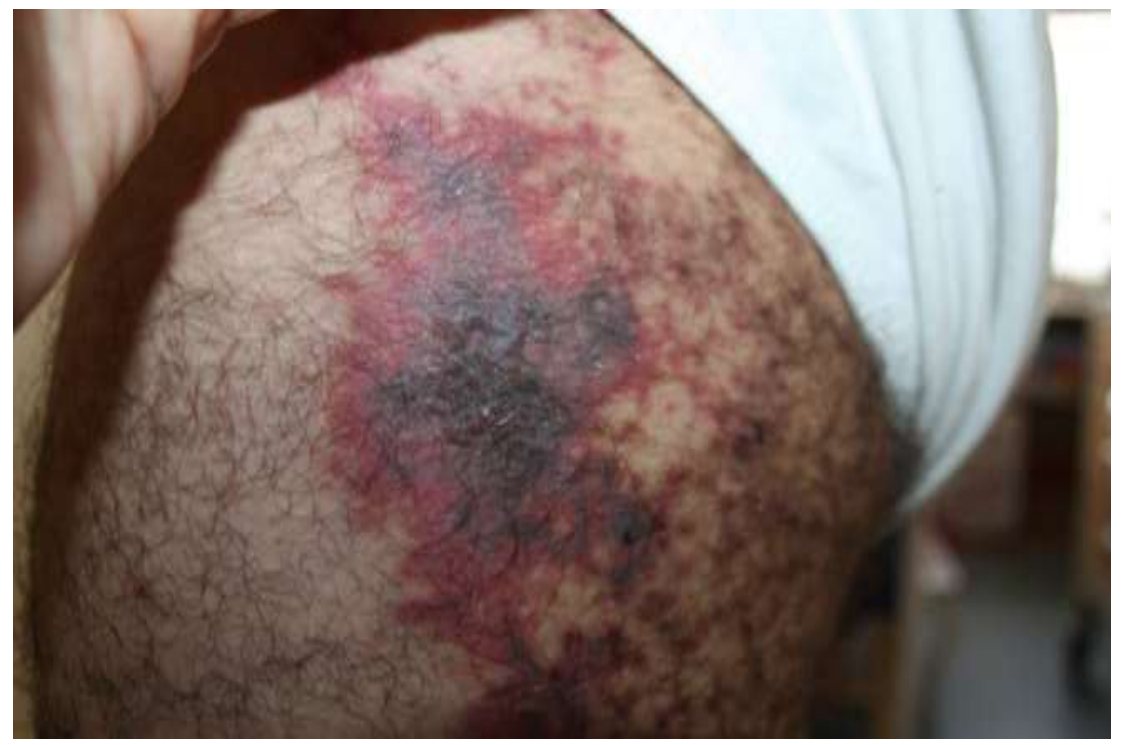

Fig 2: Purpuric lesions on the buttocks. 
Kavala et al

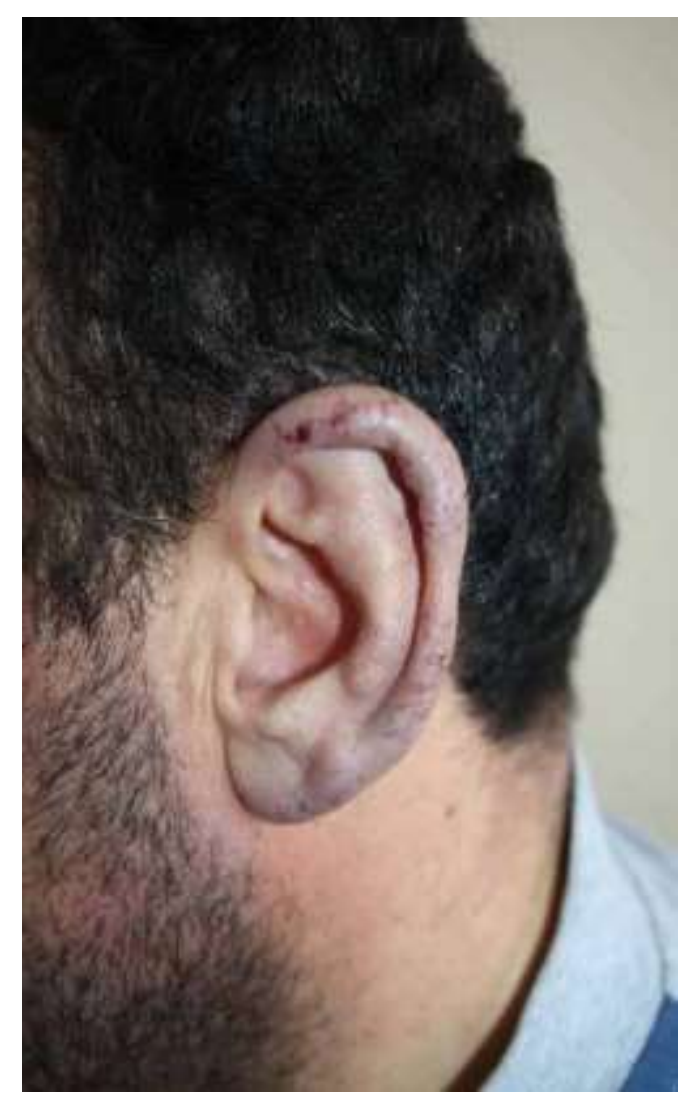

Fig 3: Similar morphological lesions in his ears. 


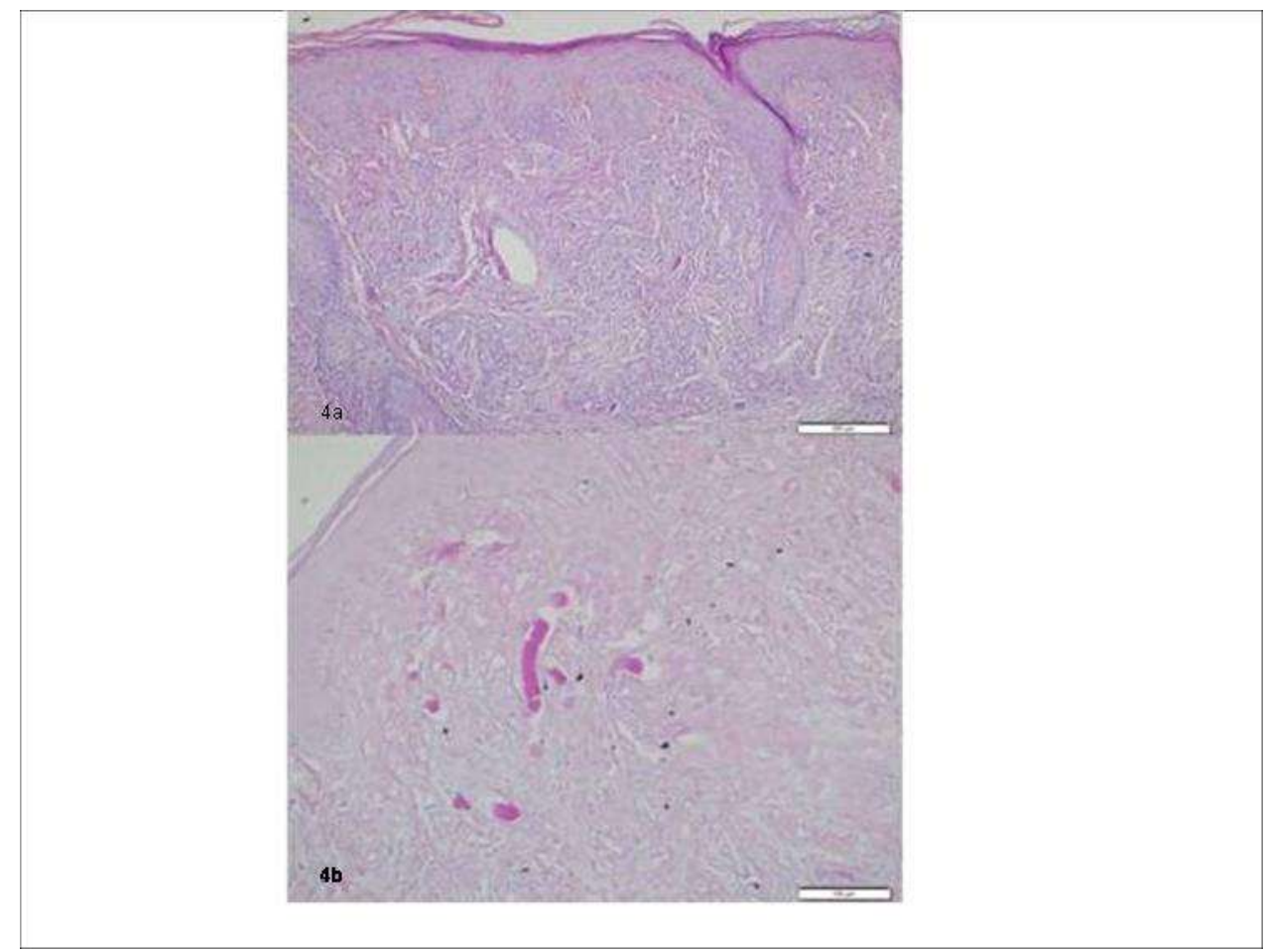

Fig 4: (a) Vascular dilatation and accumulation of eosinophilic material in lumen of vessels (H\&E, x10), (b) Eosinophilic material in lumen of vessels (PAS,x10)

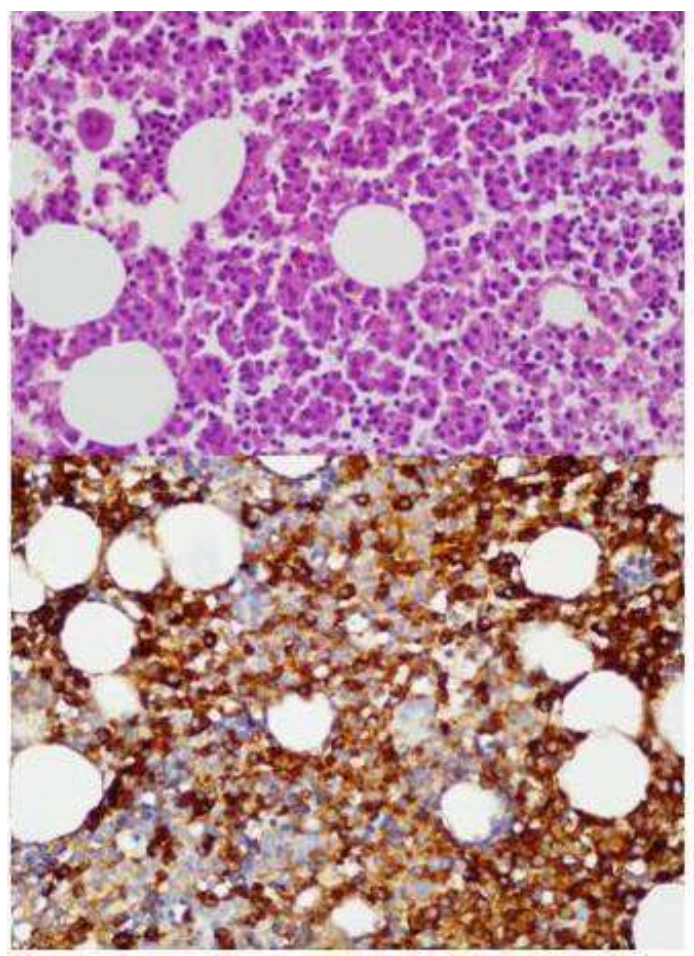

Fig.5: (a) Plasma cell infiltration in bone marrow (H\&E, x20), (b) Plasma cell stained with kappa antibody (KA, x20). 